\title{
Diagnostic value of diffusion-weighted imaging with synthetic b-values in breast tumors: comparison with dynamic contrast-enhanced and multiparametric MRI
}

\author{
Isaac Daimiel Naranjo ${ }^{1}$. Roberto Lo Gullo ${ }^{1,2}$ - Carolina Saccarelli ${ }^{1}$. Sunitha B. Thakur ${ }^{1,3}$ - Almir Bitencourt ${ }^{1,4}$. \\ Elizabeth A. Morris ${ }^{1} \cdot$ Maxine S. Jochelson ${ }^{1} \cdot$ Varadan Sevilimedu $^{5} \cdot$ Danny F. Martinez ${ }^{1} \cdot$ Katja Pinker-Domenig $^{1,6} \mathbb{C}_{\mathbb{C}}$
}

Received: 23 January 2020 / Revised: 22 May 2020 / Accepted: 21 July 2020 / Published online: 11 August 2020

(C) The Author(s) 2020

\begin{abstract}
Objectives To assess DWI for tumor visibility and breast cancer detection by the addition of different synthetic b-values.

Methods Eighty-four consecutive women who underwent a breast-multiparametric-MRI (mpMRI) with enhancing lesions on DCE-MRI (BI-RADS 2-5) were included in this IRB-approved retrospective study from September 2018 to March 2019. Three readers evaluated DW acquired b-800 and synthetic b-1000, b-1200, b-1500, and b-1800 s/mm images for lesion visibility and preferred b-value based on lesion conspicuity. Image quality (1-3 scores) and breast composition (BI-RADS) were also recorded. Diagnostic parameters for DWI were determined using a 1-5 malignancy score based on qualitative imaging parameters (acquired + preferred synthetic b-values) and ADC values. BI-RADS classification was used for DCE-MRI and quantitative ADC values + BI-RADS were used for mpMRI.

Results Sixty-four malignant (average $=23 \mathrm{~mm}$ ) and 39 benign (average $=8 \mathrm{~mm}$ ) lesions were found in $80 \mathrm{women}$. Although $\mathrm{b}$ 800 achieved the best image quality score, synthetic b-values $1200-1500 \mathrm{~s} / \mathrm{mm}^{2}$ were preferred for lesion conspicuity, especially in dense breast. b-800 and synthetic b-1000/b-1200 s/mm values allowed the visualization of $84-90 \%$ of cancers visible with DCE-MRI performing better than b-1500/b-1800 s/mm ${ }^{2}$. DWI was more specific $(86.3 \%$ vs $65.7 \%, p<0.001)$ but less sensitive $(62.8 \%$ vs $90 \%, p<0.001)$ and accurate $(71 \%$ vs $80.7 \%, p=0.003)$ than DCE-MRI for breast cancer detection, where mpMRI was the most accurate modality accounting for less false positive cases.

Conclusion The addition of synthetic b-values enhances tumor conspicuity and could potentially improve tumor visualization particularly in dense breast. However, its supportive role for DWI breast cancer detection is still not definite.

Key Points

- The addition of synthetic b-values (1200-1500 s/mm $\mathrm{mm}^{2}$ to acquired DWI afforded a better lesion conspicuity without increasing acquisition time and was particularly useful in dense breasts.

- Despite the use of synthetic b-values, DWI was less sensitive and accurate than DCE-MRI for breast cancer detection.

- A multiparametric MRI modality still remains the best approach having the highest accuracy for breast cancer detection and thus reducing the number of unnecessary biopsies.
\end{abstract}

Electronic supplementary material The online version of this article (https://doi.org/10.1007/s00330-020-07094-z) contains supplementary material, which is available to authorized users.

Katja Pinker-Domenig

pinkerdk@mskcc.org; katja.pinker-domenig@meduniwien.ac.at

1 Department of Radiology, Breast Imaging Service, Memorial Sloan Kettering Cancer Center, 300 E 66th Street, New York, NY 10065, USA

2 Department of Radiology, Breast Imaging Division, Istituto Europeo di Oncologia, Via Giuseppe Ripamonti, 435, 20141 Milano, Italy

3 Department of Medical Physics, Memorial Sloan Kettering Cancer Center, 1275 York Ave, New York, NY 10065, USA
4 Department of Imaging, A.C.Camargo Cancer Center, SP São Paulo, Brazil

5 Department of Epidemiology and Biostatistics, Memorial Sloan Kettering Cancer Center, 1275 York Ave, New York, NY 10065, USA

6 Department of Biomedical Imaging and Image-guided Therapy Division of Molecular and Gender Imaging, Medical University of Vienna, Waehringer Guertel 18-20, 1090 Vienna, Austria 
Keywords Breast tumors · Image analysis · Diagnostic imaging · Diffusion magnetic resonance imaging · Echo-planar imaging

$\begin{array}{ll}\text { Abbreviations } \\ \text { ADC } & \begin{array}{l}\text { Apparent diffusion } \\ \text { coefficient }\end{array} \\ \text { AUC } & \begin{array}{l}\text { Area under the ROC curve } \\ \text { CI }\end{array} \\ \text { Confidence interval } \\ \text { DCIS } & \text { Ductal carcinomas in situ } \\ & \begin{array}{l}\text { Diffusion-weighted } \\ \text { imaging }\end{array} \\ \text { EPI } & \text { Echo-planar imaging } \\ \text { FGT } & \text { Fibroglandular tissue } \\ \text { IDC } & \text { Invasive ductal carcinoma } \\ \text { ILC } & \text { Invasive lobular } \\ & \text { carcinoma } \\ \text { NME } & \text { Non-mass enhancing } \\ \text { NPV } & \text { Negative predictive value } \\ \text { PPV } & \text { Positive predictive value } \\ \text { ROC } & \text { Receiver operating curve } \\ \text { ROI } & \text { Region of interest } \\ \text { STIR } & \text { Short TI } \\ & \text { inversion-recovery }\end{array}$

\section{Introduction}

Diffusion-weighted imaging (DWI) is increasingly incorporated into breast MRI protocols worldwide [1-3]. DWI using apparent diffusion coefficient (ADC) mapping has reported sensitivities of up to $96 \%$ and specificities of up to $100 \%$ for breast cancer detection $[4,5]$. Currently, the prime focus of DWI is to differentiate between benign and malignant lesions to prevent unnecessary breast biopsies. With the recent concerns regarding the safety of gadolinium-based contrast agents (GBCAs) [6-8], DWI has been proposed as a promising alternative to dynamic contrast-enhanced magnetic resonance imaging (DCE-MRI) to detect early breast cancer without the costs and safety concerns associated with GBCAs [9-14].

Several studies have demonstrated that the sensitivity of unenhanced MRI with DWI was equal to or superior to mammography [4, 15]; however, there is still room for improvement [16]. Diffusion sensitivity, better known as "b-value," has important implications for tumor conspicuity and can be controlled by modifying the magnitude and duration of the diffusion gradients. Higher b-values seem to improve lesion conspicuity by suppressing the normal breast tissue and decreasing the T2 shine-through effect [17]. Nevertheless, they require long examination times and the image quality may be compromised due to a low signal-to-noise ratio [18]. Synthetic b-values may overcome these limitations. Synthetic b-values are generated through a mathematical computation technique from at least two different lower b-values in a voxelwise manner [19-21] without increasing the scan time or reducing the image quality (in fact, synthetic b-values present a higher image quality than the acquired b-values) [22] and therefore have the potential to improve the sensitivity of breast cancer detection.

The aim of our study was to assess lesion visibility and the diagnostic performance of DWI for breast cancer detection by the addition of different synthetic b-values.

\section{Materials and methods}

\section{Patients}

This single-institution study and retrospective data analysis was approved by the Institutional Review Board and was conducted in compliance with the Health Insurance Portability and Accountability Act.

Between September 2018 and March 2019, 84 consecutive women who underwent a breast MRI examination (including DCE-MRI and DWI) at our institution and fulfilled the inclusion criterion of presenting with an enhancing lesion on DCEMRI (categories 2-5 of the Breast Imaging Reporting and Data System (BI-RADS)) were included in this study. Indications for an MRI examination in these women included screening $(46.2 \%)$, extent of the disease and surgical planning $(33.8 \%)$, inconclusive findings in other imaging modalities (6.2\%), MRI follow-up examinations for previous findings $(5 \%)$, evaluation of recurrent tumor $(6.3 \%)$, and nipple discharge $(2.5 \%)$. Patients undergoing chemotherapy; pregnant women; and those undergoing examinations without DWI series, a biopsy-proven histology, or at least lesion stability for 24 months were excluded.

Due to technical failure of the DWI sequence and the presence of a clip/biopsy change generating obvious image distortion, four patients were excluded, resulting in a final study population of 80 women (mean age $48.1 \pm 12.5$ years; range 26-76 years) with 103 breast lesions. Forty-five of these patients were premenopausal (56.25\%) and 35 were post-menopausal $(43.75 \%)$.

\section{MRI examination}

All the examinations were performed using a 3-T MRI scanner (Discovery MR750; GE Healthcare) with a dedicated 16channel phased-array breast coil (Sentinelle Coil, Hologic). All the women underwent a state-of-the-art multiparametric MRI (mpMRI) protocol with T2-weighted imaging, DCEMRI, and DWI. DW images were always acquired before contrast agent injection using a single-shot echo-planar imaging (EPI) sequence with 0 and $800 \mathrm{~s} / \mathrm{mm}^{2} \mathrm{~b}$-values (Supplemental Table 1, Supplemental Digital Content 1). Synthetic DWI b-values 1000, 1200, 1500, and $1800 \mathrm{~s} / \mathrm{mm}^{2}$ 
were automatically generated from the acquired $b$-values using a built-in software. Synthetic b-values were selected based on previous literature $[23,24]$.

\section{Image analysis}

Three dedicated breast radiologists (I.D., R.L., and C.S.) with 4-5 years of experience in interpretation of multiparametric breast MRI evaluated images independently using OsiriX v.9.0 software (OsiriX). Readers were aware of the presence of lesions in all the examinations but were blinded to any clinical information and conventional and prior imaging.

\section{DWI}

Readers first assessed DW images (b-800, b-1000, b-1200, b1500 , and b-1800 s/mm ${ }^{2}$ and ADC maps blinded to the DCEMRI. For all the lesions, visibility using each b-value (yes/no), location, and laterality were recorded. If more than one lesion was visible, all lesions were recorded. A visual grading image quality score ( 1 = bad quality, $2=$ average, $3=$ good quality $)$ was assigned by each reader for all the b-values based on artifacts and fat suppression. In addition, a preferred b-value was selected by each reader based on lesion conspicuity defined as the visual difference in lesion contrast with the surrounding parenchyma.

One 2D region of interest (ROI) per lesion and reader was drawn manually on ADC maps derived from acquired b-values using the OsiriX v.9.0 software (OsiriX). The ROI was placed in a slice containing the tumor maximum diameter and within the area with the lowest ADC values.

Each reader assigned a 1-5 malignancy score to DW images (from $1=$ non-suspicious to $5=$ highly suspicious) using acquired and preferred synthetic b-values for each visible lesion. The criteria for this score included qualitative parameters based upon the previous literature $[15,25]$ as well as quantitative ADC values extracted from ADC maps as shown in Table 1. Scores 4 and 5 were considered suspicious for malignancy, whereas scores 1, 2, and 3 were considered non-suspicious.

Table 1 Criteria for DWI malignancy score

\begin{tabular}{lll}
\hline Descriptor & DWI + ADC map & \\
\cline { 2 - 3 } & Suspicious & Not suspicious \\
\hline Internal signal & Heterogeneous & Homogeneous \\
Shape & Irregular/angulated & Round $/$ oval \\
ADC & $\leq 1.3 \times 10^{-3} \mathrm{~mm}^{2} / \mathrm{s}$ & $>1.3 \times 10^{-3} \mathrm{~mm}^{2} / \mathrm{s}$ \\
\hline
\end{tabular}

$D W I$, diffusion-weighted image; $A D C$, apparent diffusion coefficient

\section{DCE-MRI}

After a wash-out period of at least 21 days, DCE-MRI alone was read. Readers classified lesions according to BI-RADS classification [26]. Lesions categorized as BI-RADS 2/3 were considered non-suspicious, whereas categories BIRADS 4/5 were considered suspicious for malignancy.

Consequently, the results for both readings were reviewed in consensus for missed lesions on DWI or a lesion mis-match between DCE-MRI and DWI. In the case of mis-matched or missed lesions on DWI by one or two of the readers, they were asked to obtain $\mathrm{ADC}$ values for lesion categorization. Lesions missed by all the readers were excluded for categorization. The mean ADC values for all the lesions across readers were then determined (Supplemental Table 2, Supplemental Digital Content 1). Categories for breast composition of fibroglandular tissue (FGT) were recorded for each examination based on its report (A-almost entirely fat, B-scattered FGT, C-heterogeneous FGT, and D-extreme FGT).

\section{Multiparametric MRI}

mpMRI with DWI and DCE-MRI was evaluated using an ADC cutoff value of $1.3 \times 10^{-3} \mathrm{~mm}^{2} / \mathrm{s}$ as recommended by the European Society of Breast Imaging [15]. A final lesion classification was given as follows: If a BI-RADS 4 or 5 was assigned on DCE-MRI, an ADC $>1.3 \times 10^{-3} \mathrm{~mm}^{2} / \mathrm{s}$ was required to assign a final classification as non-suspicious. If a BIRADS 2 or 3 was assigned, an $\mathrm{ADC} \leq 1.3 \times 10^{-3} \mathrm{~mm}^{2} / \mathrm{s}$ was required to assign a final classification as suspicious.

\section{Histopathology}

The final diagnosis was established by histopathology using image-guided needle biopsy for the majority of the lesions ( $n=98)$. In the event of discordant findings between histopathology and imaging, the final diagnosis was established surgically $(n=2)$. Benignity was confirmed in three lesions by imaging follow-up of up to 24 months.

\section{Statistical analysis}

All calculations were performed using SPSS 25.0 (IBM) and SAS 9.4 (SAS Institute) in a per-lesion analysis. Median and mean ranks were calculated for image quality and preferred bvalues. Sensitivity, specificity accuracy, and their $95 \%$ confidence intervals $(\mathrm{CI})$ were calculated for the imaging methods and averaged over the three readers [27]. Likewise, diagnostic parameters for breast cancer detection were obtained for each imaging modality for lesions stratified by size (small lesions $\leq 10 \mathrm{~mm}$ and lesions $>1 \mathrm{~mm}$ ). Receiver operating curves (ROC) were obtained using the PROC GLIMMIX statement in SAS 9.4 (SAS Institute) by treating each reader's assessment 
as a fixed effect and estimating a robust (sandwich) measure of variance to account for the correlation between multiple readers [28]. The epidemiological parameters and the areas under the ROC curves (AUC) were compared between the three diagnostic modalities by using chi-square tests [29]. Bonferroni's correction was made for multiple pairwise comparisons $(\alpha=0.016)$.

Cohen's $\kappa$ and concordance correlation coefficient statistics were used to determine the concordance between imaging methods and readers [30]. The inter-reader agreement between ADC measurements was documented using BlandAltman plots where the ADC mean was used.

\section{Results}

One hundred and three lesions (91 enhancing masses and 12 non-mass lesions (NMLE)) from 80 women were assessed including 64 malignant tumors (mean size $23 \mathrm{~mm}$; range 5$100 \mathrm{~mm}$ ) and 39 benign lesions (mean size $8 \mathrm{~mm}$; range 5$22 \mathrm{~mm}$ ). Histological results are shown in Table 2. Breast composition of these women included 37 (46.2\%) women within categories $\mathrm{A} / \mathrm{B}$ and $43(53.8 \%)$ women within categories $\mathrm{C} / \mathrm{D}$.

\section{Visual grading image quality score}

Acquired b- $800 \mathrm{~s} / \mathrm{mm}^{2}$ was the best rated by all the readers in terms of image quality, whereas synthetic b-1800 $\mathrm{s} / \mathrm{mm}^{2}$ was the worst rated. Mean image quality scores for the different bvalues are summarized in Supplemental Table 3 in the Electronic Supplementary Material.

\section{Overall lesion visibility with DWI}

No mis-matched lesions with DCE-MRI were identified. The percentage of enhancing lesions identified on DWI was $82.5 \%$ for reader $1(\mathrm{r} 1), 78.6 \%$ for reader $2(\mathrm{r} 2)$, and $81.5 \%$ for reader 3 (r3). DWI visualized $84-90 \%$ of the malignant enhancing lesions. Results of missed lesions by reader are summarized in Table 3 . Readers missed the same number of malignant lesions in acquired b-800 $\mathrm{s} / \mathrm{mm}^{2}$ and synthetic b-1000/b-1200 s/mm² images (r1: 8, r2: 10, r3: 6). Fewer tumors were visualized at higher b-values b-1500 (r1: 10, r2: 12, r3: 6) and b-1800 s/mm² (r1: 11, r2: 14, r3: 11).

DWI performed poorly for lesion visibility of small tumors $(<10 \mathrm{~mm})$ and benign lesions, especially synthetic $b-$ $1800 \mathrm{~s} / \mathrm{mm}^{2}$ values. Results for missed lesions stratified by reader and b-values are summarized in Supplemental Table 4 in the Electronic Supplementary Material. Figure 1 shows an example of a benign lesion not seen by extremely high b-values.

Among the lesions missed by all the readers (10 enhancing lesions representing 9.7\%), one 8-mm malignant NMLE was not visible on DWI due to being included in the gap between slices in DWI. The remaining nine lesions included 4 NMLE (mean size $11.7 \mathrm{~mm}$; range 6-20 mm), of which two were $<10 \mathrm{~mm}$ and 5 masses $<10 \mathrm{~mm}$ (mean size $5.6 \mathrm{~mm}$; range $5-8 \mathrm{~mm}$ ) with an overall mean lesion size of $8.3 \mathrm{~mm}$. Histology of missed tumors was benign in five cases (one benign breast parenchyma (NMLE), one papilloma (mass), two fibroadenomas (masses), and one unchanged lesion on follow-up (mass)) and malignant in four cases (two ductal carcinomas in situ (DCIS) (NMLE), one invasive ductal carcinoma (IDC) grade I (mass), and one

Table 2 Histopathology of all lesions stratified by benignity and malignancy

\begin{tabular}{|c|c|c|c|c|}
\hline \multicolumn{2}{|l|}{ Benign lesions $(n=39)(37.9 \%)$} & \multicolumn{3}{|c|}{ Malignant lesions $(n=64)(62.1 \%)$} \\
\hline Fibroadenoma & $16(15.6 \%)$ & IDC & Total & $53(51.5 \%)$ \\
\hline Fibrocystic changes & $4(3.9 \%)$ & & Low grade & $4(3.9 \%)$ \\
\hline \multirow[t]{3}{*}{ Benign breast parenchyma } & $2(1.9 \%)$ & & Intermediate grade & $25(24.3 \%)$ \\
\hline & & & High grade & $24(23.3 \%)$ \\
\hline & & & DCIS associated & $12(22.6 \%)$ \\
\hline ALH & $1(0.9 \%)$ & ILC & & $5(4.8 \%)$ \\
\hline PASH & $5(4.9 \%)$ & & & \\
\hline Papilloma & $2(1.9 \%)$ & & & \\
\hline $\mathrm{ADH}$ & $1(0.9 \%)$ & DCIS & & $6(5.8 \%)$ \\
\hline Hamartoma & $1(0.9 \%)$ & & & \\
\hline Benign unchanged (at least 2-year follow-up) & $3(2.9 \%)$ & & & \\
\hline Stromal fibrosis and adenosis & $3(2.9 \%)$ & & & \\
\hline LCIS & $1(0.9 \%)$ & & & \\
\hline
\end{tabular}

$A L H$, atypical lobular hyperplasia; $P A S H$, pseudoangiomatous hyperplasia; $A D H$, atypical ductal hyperplasia; $I D C$, invasive ductal carcinoma; $I L C$, invasive lobular carcinoma; DCIS, ductal carcinoma in situ; LCIS, lobular carcinoma in situ 
Table 3 Lesions not visible with DWI for all readers

\begin{tabular}{lcccc}
\hline Histology & Reader 1 & Reader 2 & Reader 3 & Lesions missed by all readers \\
\hline Benign & 10 & 12 & 13 & 5 \\
Malignant & 8 & 10 & 6 & 5 \\
Total of missed lesions & $18(17.5 \%)$ & $22(21.4 \%)$ & $19(18.5 \%)$ & $10(9.7 \%)$ \\
Mean lesion size (range) & $7.7 \mathrm{~mm}(5-20 \mathrm{~mm})$ & $8.7 \mathrm{~mm}(5-22 \mathrm{~mm})$ & $7.9 \mathrm{~mm}(5-20 \mathrm{~mm})$ & $8.3 \mathrm{~mm}(5-20 \mathrm{~mm})$ \\
$\%$ of lesions visualized with DWI & $82.5 \%$ & $78.6 \%$ & $81.5 \%$ & \\
\hline
\end{tabular}

invasive lobular carcinoma (ILC) (NMLE)). Figure 2 shows an example of a missed lesion by all the readers.

\section{Preferred synthetic b-value}

Synthetic b-values $1200-1500 \mathrm{~s} / \mathrm{mm}^{2}$ provided the best lesion conspicuity across all readers. Reader 1 preferred b-1500 $\mathrm{s} / \mathrm{mm}^{2}$, while readers 2 and 3 preferred b-1200 $\mathrm{s} / \mathrm{mm}^{2}$. Benign lesions were more conspicuous at lower b-values, while malignant tumors appeared brighter than the surrounding parenchyma at high b-values (Fig. 3). Readers preferred lower b-values for benign lesions (r1: b-1000; r2: b-800; and $\mathrm{r} 3: \mathrm{b}-1200 \mathrm{~s} / \mathrm{mm}^{2}$ ) and higher b-values for malignant tumors (r1: b-1500; r2: b-1200; and r3: b-1500 s/mm $\mathrm{mm}^{2}$. This was particularly relevant in relation to the amount of FGT. Twenty-nine malignant lesions were found within breast composition categories C/D. In this subgroup, readers preferred higher b-values (r1: b-1500; r2: b-1200; and r3: b-1500 s/mm ${ }^{2}$.

\section{Breast cancer detection}

Diagnostic parameters for DWI, DCE-MRI, and mpMRI are summarized in Table 4.

\section{DWI and DCE-MRI}

DWI was significantly less sensitive than DCE-MRI (62.8\% vs $90 \%, p<0.0001)$. However, DWI was significantly more specific than DCE-MRI (86.3\% vs $65.7 \%$, $p<0.0001)$. DWI detected fewer cancers than DCEMRI, with 21 (reader 1) and 23 (readers 2 and 3) false
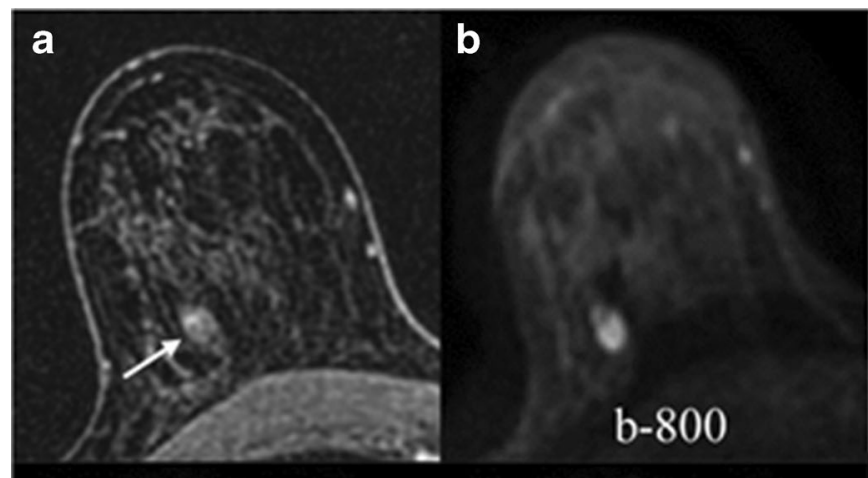

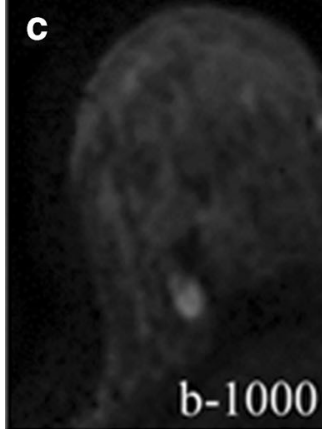

Fig. 1 Axial contrast-enhanced (a), acquired DWI (b), and synthetic DWI (c) images of a patient with a $10-\mathrm{mm}$ biopsy-proven fibroadenoma in the third posterior depth of the right breast. This benign lesion is less conspicuous at higher b-values being barely visible at b- 1500 and b-1800 s/mm ${ }^{2}$. ADC mean value for this lesion was $1850 \times$
$10^{-6} \mathrm{~mm}^{2} / \mathrm{s}$. This lesion was correctly categorized as non-suspicious in both DWI and DCE-MRI. DWI, diffusion-weighted imaging; ADC, apparent diffusion coefficient; DCE-MRI, dynamic contrast-enhanced magnetic resonance imaging 


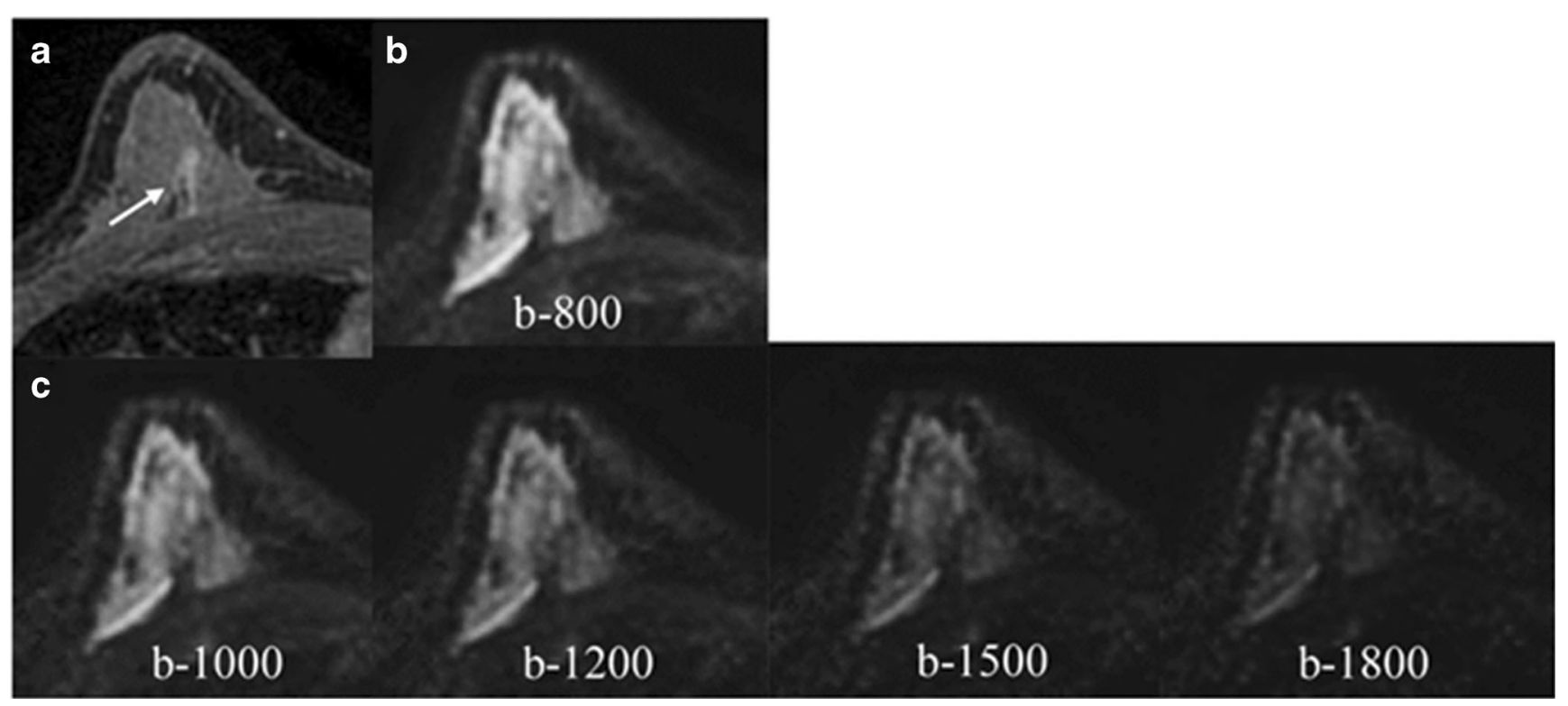

Fig. 2 Axial images of a biopsy-proven DCIS in a patient with dense breast. Contrast-enhanced image (a) shows a 14-mm non-mass enhancement in the posterior third of the right breast (arrow) in the middle third. All readers missed this lesion on DWI which was

negative cases on DWI (Supplemental Table 5, Electronic Supplementary Material). Figure 4 shows a case of a false negative on DWI. DWI had a significantly lower diagnostic accuracy for breast cancer detection ( $71 \%$ vs $80.7 \%$, $p=0.003)$. When comparing the diagnostic performance of DWI and DCE-MRI, DWI achieved a lower AUC (0.82 vs $0.89, p=0.14$ ). indistinguishable either in the acquire DWI b-800 (b) or the synthetic bvalues (c). DCIS, ductal carcinoma in situ; DWI, diffusion-weighted imaging

\section{Multiparametric MRI}

mpMRI using DCE-MRI and DWI significantly maximized the sensitivity compared with using DWI alone $(95.5 \%$ vs $62.8 \%, p<0.0001)$ but was not significantly different than DCE-MRI $(95.5 \%$ vs $90 \%, p=0.02)$. mpMRI maintained a high specificity compared with DWI alone $(75.5 \%$ vs $86.3 \%$,

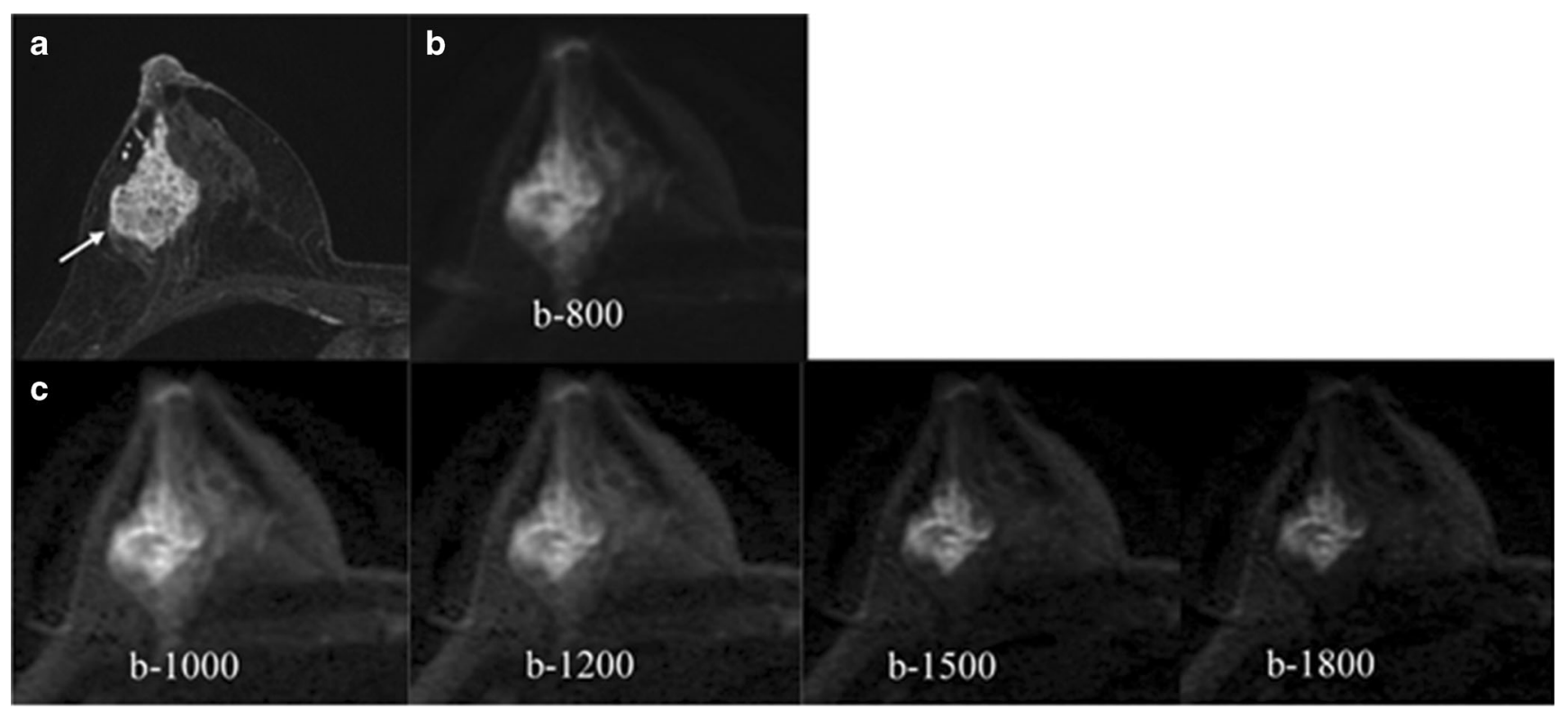

Fig. 3 Axial images of a 47-year-old woman with dense breasts. Contrast-enhanced image (a) shows a 52-mm NMLE in the outer quadrants of the right breast. Biopsy proved a high-grade invasive ductal carcinoma. DWI acquired image (b) and synthetic images (c) are depicted. An increased lesion-to-normal-tissue contrast at synthetic high $\mathrm{b}$-values due to suppression of the glandular signal is noticed. ADC mean value for this lesion was $1266 \times 10^{-6} \mathrm{~mm}^{2} / \mathrm{s}$. This lesion was correctly categorized as suspicious in both DWI and DCE-MRI. NMLE, non-mass lesion; DWI, diffusion-weighted imaging; ADC, apparent diffusion coefficient; DCE-MRI, dynamic contrast-enhanced magnetic resonance imaging 


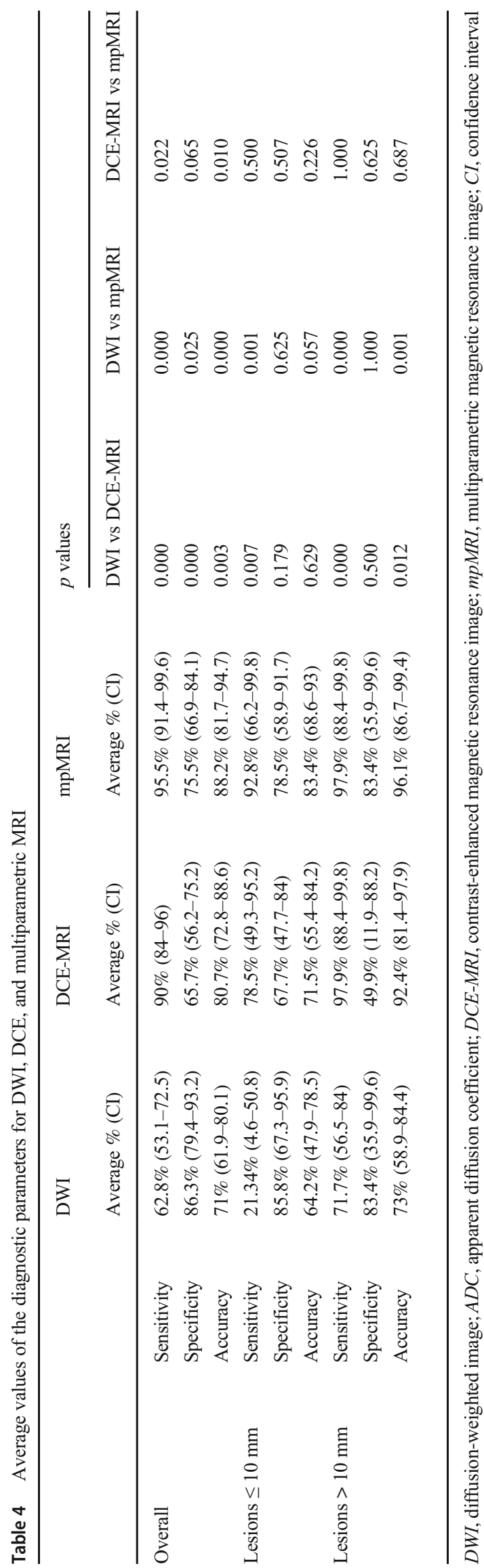

$p=0.02)$. mpMRI was significantly more accurate than DWI ( $88.2 \%$ vs $71 \%, p<0.0001)$ and DCE-MRI $(88.2 \%$ vs $80.7 \%$, $p=0.010$ ) across all readers. When comparing the diagnostic performance of mpMRI with DWI and DCE-MRI, mpMRI achieved a better AUC than DWI ( 0.92 vs $0.82, p=0.05)$ and DCE-MRI ( 0.92 vs $0.89, p=0.64)$, although these differences were not significant. Figure 5 shows ROC curves comparing DWI, DCE-MRI, and mpMRI.

When stratifying lesions by size, DWI was less accurate for lesions $\leq 10 \mathrm{~mm}$ than for lesions over $10 \mathrm{~mm}(64.2 \%$ vs $73 \%)$. Although mpMRI showed better accuracy than DCE-MRI in both subgroups, a significant difference was achieved only when both groups were considered. The number of false positives with mpMRI was consistently lower than with DCEMRI in both groups, with a higher reduction in the group of lesions $\leq 10 \mathrm{~mm}$. Results are shown in Supplemental Table 6 in the Electronic Supplementary Material. All misclassified benign lesions on DCE-MRI which were correctly classified by the addition of ADC values were BI-RADS 4 except two cases classified as BI-RADS 5 by reader 2 (one $13 \mathrm{~mm}$ fibroadenoma and one $7 \mathrm{~mm}$ NMLE pseudoangiomatous hyperplasia in a patient with a contralateral cancer).

\section{Inter-reader agreement}

DWI inter-reader agreement was moderate to high for lesion visibility ( $\kappa=0.41-0.63)$, preferred $b$-value $(\kappa=0.47-0.56$ ), DWI malignancy score $(\kappa=0.63-0.76)$, and DCE-MRI assigned BI-RADS $(\kappa=0.61-0.65)$. Inter-reader agreement for image quality ranged from fair to high $(\kappa=0.30-0.86)$ across all $b$-values. The highest level of inter-reader agreement was for $\mathrm{b}-800 \mathrm{~s} / \mathrm{mm}^{2}$ (average $\kappa=0.65$ ), whereas the lowest $\kappa$ value was for b-1800 s/mm (average $\kappa=0.52$ ). Inter-reader agreement for image quality of the intermediate b-values was similar across all readers (average $\kappa=0.58$ ). Details for inter-reader agreement are shown in Table 5. ADC achieved a high to almost perfect agreement between readers (rho $\_c=0.90-0.73$ ) (shown in Fig. 6).

\section{Discussion}

DWI has been proposed as an unenhanced option for breast cancer screening by MRI. Synthetic b-values may improve lesion visibility without increasing the acquisition time while avoiding the disadvantages of performing DWI at very high bvalues (i.e., eddy current distortions). In this study, we assessed DWI for tumor visibility and breast cancer detection by a combination of acquired $b$-values $\left(800 \mathrm{~s} / \mathrm{mm}^{2}\right)$, its ADC maps, and different synthetic b-values $(1000,1200,1500$, and $1800 \mathrm{~s} / \mathrm{mm}^{2}$ ). Acquired b-800 and synthetic b-1000/1200 $\mathrm{s} / \mathrm{mm}^{2}$ values allowed the visualization of $84-90 \%$ of the malignant enhancing tumors in this study. Image quality score 


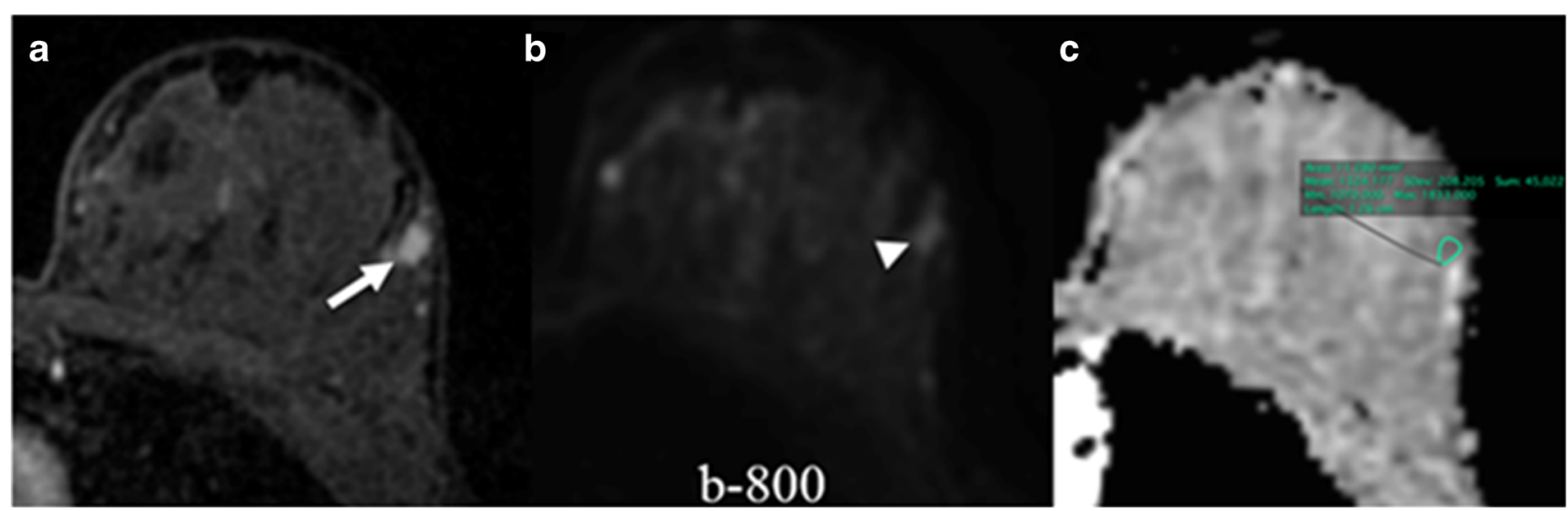

Fig. 4 A case of a false negative in DWI. Axial images of a biopsyproven DCIS. Contrast-enhanced image (a) shows a 7-mm oval irregular enhancing lesion (arrow) in the left breast. Acquired DWI b800 (b) shows an oval apparently restricting lesion (arrowhead). The ADC map (c) shows a region of interest drawn in the corresponding

was lower for b-1500/1800 s/mm ${ }^{2}$ values; these values (especially the latter value) missed a higher number of lesions. Synthetic b-values of $1200-1500 \mathrm{~s} / \mathrm{mm}^{2}$ provided the best lesion conspicuity. Benign lesions were more conspicuous at lower b-values, while malignant tumors appeared brighter than the surrounding parenchyma at higher b-values, especially in breast composition categories C/D. Despite the use of synthetic b-values, DWI was less sensitive and accurate than DCE-MRI for breast cancer detection. mpMRI maintained an excellent sensitivity and a high specificity compared with DCE-MRI and DWI alone and, therefore, significantly increased the accuracy compared with both modalities separately.

In this study, we showed that $78-82 \%$ of the lesions visible with DCE-MRI were visualized with DWI alone including both benign and malignant breast lesions. Among those lesions missed, the majority of them were either small $(\leq 10$ $\mathrm{mm}$ ) or NMLE. This is in agreement with prior studies investigating DWI with ADC mapping that showed that lesions location which yields an ADC mean value of $1324 \times 10^{-6} \mathrm{~mm}^{2} / \mathrm{s}$. This lesion was correctly categorized as malignant in DCE-MRI but categorized as benign in DWI. DWI, diffusion-weighted imaging; DCIS, ductal carcinoma in situ; ADC, apparent diffusion coefficient; DCE-MRI, dynamic contrast-enhanced magnetic resonance imaging

missed with DWI were either small or NMLE [16, 31, 32]. This is potentially problematic if one of the future roles of DWI is to be a reliable tool in breast cancer detection and not only in the characterization of lesions found in other imaging modalities. An improvement in the resolution of DWI sequence would be desirable to enhance cancer detection. Regarding synthetic b-values, readers were able to identify the same number of cancers using synthetic b-values of $1000 / 1200 \mathrm{~s} / \mathrm{mm}^{2}$ and the acquired b-value of $800 \mathrm{~s} / \mathrm{mm}^{2}$. In contrast, synthetic b-values of 1500 and $1800 \mathrm{~s} / \mathrm{mm}^{2}$ missed more lesions, probably due to a reduction in image quality.

Most of the studies have almost exclusively focused on the visibility of breast cancer [20,22,33-36]; therefore, there is limited information on the conspicuity of benign breast lesions at high or synthetic b-values. While Chen et al [37] found no significant differences in conspicuity grades using b-values of 600,800 , and $1000 \mathrm{~s} / \mathrm{mm}^{2}$, our results point to a difference in conspicuity. Benign lesions were more conspicuous at lower b-values, while malignant tumors appeared brighter than the
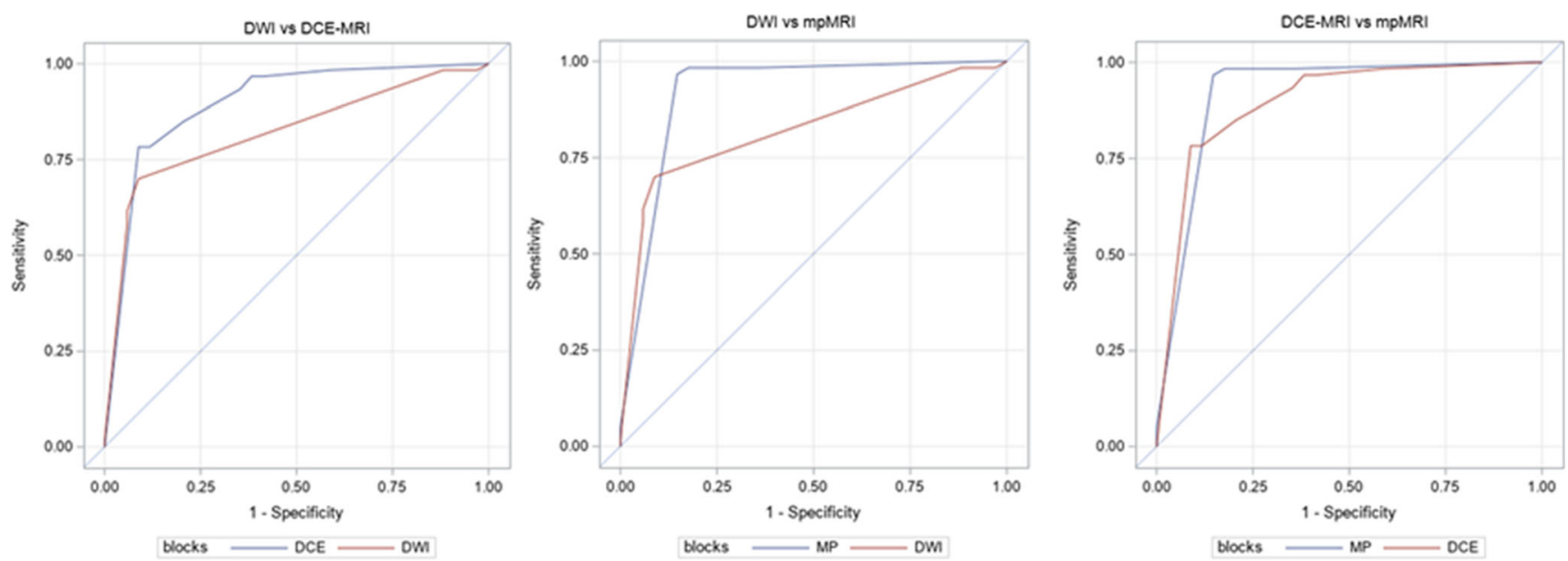

Fig. 5 Results of pairwise comparisons between the receiver operating curves (ROCs) and the area under the curve (AUC) for each diagnostic modality 
Table 5 Inter-reader agreement (weighted $\kappa$ values) for diffusion-weighted images (DWI) and dynamic contrast-enhanced MR images (DCE-MRI) parameters

\begin{tabular}{|c|c|c|c|c|c|c|}
\hline & & & r1 vs r2 & r1 vs r3 & $\mathrm{r} 2$ vs r 3 & Average \\
\hline \multirow[t]{13}{*}{ DWI } & \multirow[t]{2}{*}{$\mathrm{b}-800 \mathrm{~s} / \mathrm{mm}^{2}$} & Lesion visibility & 0.42 & 0.62 & 0.46 & 0.50 \\
\hline & & Image quality & 0.86 & 0.60 & 0.48 & 0.65 \\
\hline & \multirow[t]{2}{*}{$\mathrm{b}-1000 \mathrm{~s} / \mathrm{mm}^{2}$} & Lesion visibility & 0.41 & 0.62 & 0.50 & 0.51 \\
\hline & & Image quality & 0.76 & 0.52 & 0.46 & 0.58 \\
\hline & \multirow[t]{2}{*}{$\mathrm{b}-1200 \mathrm{~s} / \mathrm{mm}^{2}$} & Lesion visibility & 0.42 & 0.66 & 0.48 & 0.52 \\
\hline & & Image quality & 0.76 & 0.52 & 0.46 & 0.58 \\
\hline & \multirow[t]{2}{*}{ b-1500 s/mm ${ }^{2}$} & Lesion visibility & 0.56 & 0.5 & 0.52 & 0.53 \\
\hline & & Image quality & 0.76 & 0.52 & 0.46 & 0.58 \\
\hline & \multirow[t]{2}{*}{ b-1800 s/mm $\mathrm{mm}^{2}$} & Lesion visibility & 0.63 & 0.51 & 0.60 & 0.58 \\
\hline & & Image quality & 0.79 & 0.46 & 0.30 & 0.52 \\
\hline & \multicolumn{2}{|l|}{ Preferred b-value } & 0.47 & 0.56 & 0.47 & 0.50 \\
\hline & \multicolumn{2}{|l|}{ Malignancy score } & 0.76 & 0.63 & 0.74 & 0.71 \\
\hline & \multicolumn{2}{|l|}{ *ADC mean } & 0.90 & 0.77 & 0.73 & 0.80 \\
\hline DCE-MRI & \multicolumn{2}{|l|}{ BI-RADS } & 0.65 & 0.63 & 0.61 & 0.63 \\
\hline
\end{tabular}

*Apparent diffusion coefficient (ADC) values for reader's agreement are expressed using the rho concordant correlation coefficient

surrounding parenchyma at high b-values. The increased conspicuity of breast cancer at high b-values has been demonstrated by other studies with a wider range of $b$-values than Chen et al $[17,38]$.

An improved conspicuity of malignant tumors at high bvalues could be particularly helpful in dense breasts, where lesions can be mammographically masked by the large amount of FGT. In addition, an improvement of tumor visibility without contrast injection could improve the costeffectiveness of MRI [39]. However, extremely high b-values, i.e., b-1800 s/mm $\mathrm{mm}^{2}$, have a low signal which can cause lesions located on the fat tissue to be overlooked, especially if fat is poorly suppressed [33]. In light of our results, b-1200 s/mm could be the best option for an optimal lesion visualization with the best conspicuity, which could enhance lesion characterization by a better correlation on ADC maps and more accurate $\mathrm{ADC}$ values.
Nevertheless, it is worth mentioning that ADC (maps and values) can only be derived from acquired DW images. Synthetic high b-value images are obtained by extrapolating signals acquired at lower b-values (e.g., 0 and $800 \mathrm{~s} / \mathrm{mm}^{2}$ ), assuming a Gaussian model. However, diffusion in tissues is not Gaussian [2]. The calculation of synthetic high b-values is just a strategy to enhance contrast already present in lower bvalue images and is potentially useful to detect and depict lesions but lacks the power of non-Gaussian diffusion to characterize tissues [40].

Although synthetic b-values over $1000 \mathrm{~s} / \mathrm{mm}^{2}$ have demonstrated an improvement in tumor visualization and image quality $[19,20,22,34,35,40-43]$, DCE-MRI outperforms DWI for breast cancer visualization and detection with a higher sensitivity across all readers. This is in accordance with the current literature: DCE-MRI outperforms unenhanced MRI with or without supportive sequences for cancer visualization $[9,16]$.

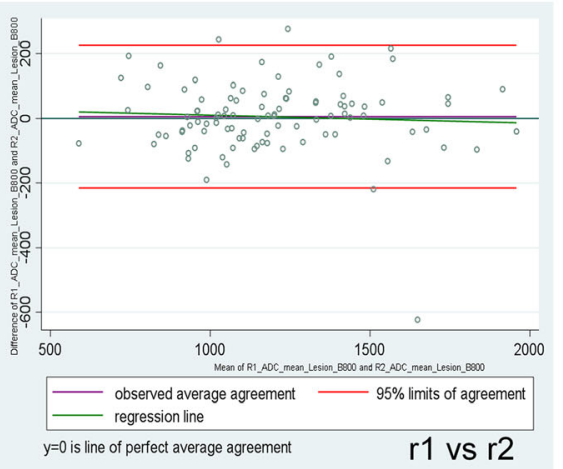

Fig. 6 Bland-Altman plots for ADC mean inter-reader agreement. Deviation of the observed data from the line of perfect concordance (line at $45^{\circ}$ on a square scatterplot) was used as a measure of
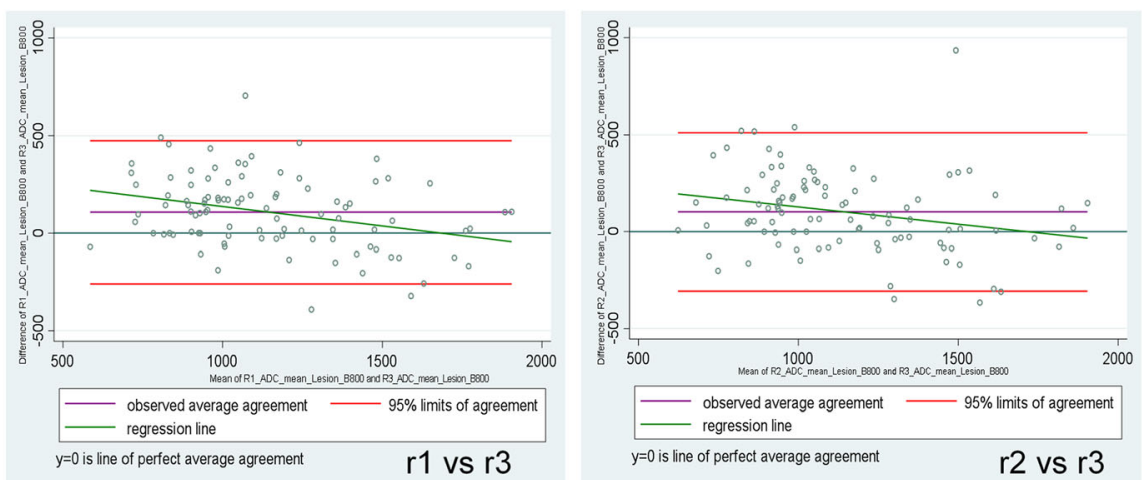

agreement. The coefficient's proximity to 1 indicates better agreement between two readers for that imaging parameter. ADC, apparent diffusion coefficient; $r$, reader 
In particular, tumors such as DCIS or NMLE exhibit a lower signal intensity in DWI and, therefore, are prone to be overlooked with unenhanced MRI, especially at high b-values [44]. These limitations are to be addressed to enable unenhanced MRI in a screening setting, where tumors tend to be smaller and NMLE lesions are clinically undetectable. In addition, these types of lesions account for false negative cases in DWI. In our study, a high number of IDC cases exhibited associated DCIS which could explain a slightly lower sensitivity for DWI compared with other studies [4]. Based on our results, DWI alone would currently have no role in the workup of indeterminate lesions (e.g., BI-RADS IVa and IVb lesions), especially in small ones where its accuracy was lower mainly at the expense of a decrease in sensitivity. In this subgroup, the sensitivity and accuracy for DCE-MRI were also reduced since there is a difficulty in distinguishing morphological features. In these cases, mpMRI continued showing the best accuracy although no significant differences with DCE-MRI were found. Nevertheless, there was an additional value in the combination of DWI and DCE-MRI: a decrease in the number of false positives. This was particularly relevant in the group of small lesions $\leq 10 \mathrm{~mm}$ which included most of the benign lesions in our study sample. This is important to prevent unnecessary follow-up examinations in indeterminate lesions as well as benign breast biopsies, which increase costs and patient anxiety.

These results match previous publications investigating a combined DWI and DCE-MRI approach for breast cancer detection [16, 45-47].

Overall, inter-reader agreement was moderate to high for all the parameters assessed. Lesion visibility at b-800 s/mm ${ }^{2}$ achieved the lowest agreement, which could point to a more consistent performance of synthetic $b$-values for lesion visibility. Inter-reader agreement was moderate for b-values rendering the best tumor conspicuity $\left(1200-1500 \mathrm{~s} / \mathrm{mm}^{2}\right.$ ). This can be explained by the fact that readers preferred a range of $b-$ values rather than a specific value. Images at b-1800 s/mm were rated worst by all readers with respect to both lesion conspicuity and image quality.

We acknowledge several limitations in our study. Firstly, no comparison was done with acquired high $b$-values to maintain clinical acquisition times. Secondly, the larger size of malignant lesions compared with the benign ones and the small number of pure DCIS, ILC, and NMLE compared with invasive carcinomas presenting with a mass may affect the results and their generalization. Nevertheless, this population reflected the clinical practice in our screening and tertiary assessment center under the established inclusion criteria. Thirdly, synthetic bvalues generated from different DWI sequences may yield different visual and image quality results. In our study, a singleshot EPI DWI with a short TI inversion-recovery (STIR) fat suppression sequence was used, and therefore, our results may not be extrapolated to other sequences.
In conclusion, the addition of synthetic high b-values (e.g., $1200 \mathrm{~s} / \mathrm{mm}^{2}$ ) improves tumor conspicuity without increasing the time of scan, which is particularly helpful in dense breasts. Nevertheless, the role of DWI for the visualization of NMLE and small lesions and its performance in breast cancer detection are still not definite. mpMRI remains the best modality for lesion detection with the best accuracy which is particularly helpful in MRI screening patients and obviates unnecessary biopsies in benign lesions.

Funding information Open access funding provided by Medical University of Vienna. This study received funding from the NIH/NCI Cancer Center Support Grant (P30 CA008748), the Breast Cancer Research Foundation, Susan G. Komen, Spanish Foundation Alfonso Martin Escudero, and the European School of Radiology.

\section{Compliance with ethical standards}

Guarantor The scientific guarantor of this publication is Katja Pinker, $\mathrm{MD}, \mathrm{PhD}$.

Conflict of interest The authors of this manuscript declare no relationships with any companies, whose products or services may be related to the subject matter of the article.

Statistics and biometry One of the authors (Varadan Sevilimedu, MBBS, DrPH) has significant statistical expertise.

Informed consent Written informed consent was waived by the Institutional Review Board.

Ethical approval Institutional Review Board approval was obtained.

Methodology
- retrospective
- observational
- performed at one institution

Open Access This article is licensed under a Creative Commons Attribution 4.0 International License, which permits use, sharing, adaptation, distribution and reproduction in any medium or format, as long as you give appropriate credit to the original author(s) and the source, provide a link to the Creative Commons licence, and indicate if changes were made. The images or other third party material in this article are included in the article's Creative Commons licence, unless indicated otherwise in a credit line to the material. If material is not included in the article's Creative Commons licence and your intended use is not permitted by statutory regulation or exceeds the permitted use, you will need to obtain permission directly from the copyright holder. To view a copy of this licence, visit http://creativecommons.org/licenses/by/4.0/.

\section{References}

1. Partridge SC, Nissan N, Rahbar H, Kitsch AE, Sigmund EE (2017) Diffusion-weighted breast MRI: Clinical applications and emerging techniques. J Magn Reson Imaging 45:337-355

2. Iima M, Honda M, Sigmund EE, Ohno Kishimoto A, Kataoka M, Togashi K (2019) Diffusion MRI of the breast: current status and 
future directions. J Magn Reson Imaging. https://doi.org/10.1002/ jmri.26908

3. Partridge SC, Newitt DC, Chenevert TL, Rosen MA, Hylton NM (2019) Diffusion-weighted MRI in multicenter trials of breast cancer. Radiology 291:546

4. Amornsiripanitch N, Bickelhaupt S, Shin HJ et al (2019) Diffusionweighted MRI for unenhanced breast cancer screening. Radiology 293:504-520

5. Chen X, Li WL, Zhang YL, Wu Q, Guo YM, Bai ZL (2010) Metaanalysis of quantitative diffusion-weighted MR imaging in the differential diagnosis of breast lesions. BMC Cancer 10:693

6. Runge VM (2017) Critical questions regarding gadolinium deposition in the brain and body after injections of the gadolinium-based contrast agents, safety, and clinical recommendations in consideration of the EMA's Pharmacovigilance and Risk Assessment Committee recommendation for suspension of the marketing authorizations for 4 linear agents. Invest Radiol 52:317-323

7. Dekkers IA, Roos R, van der Molen AJ (2018) Gadolinium retention after administration of contrast agents based on linear chelators and the recommendations of the European Medicines Agency. Eur Radiol 28:1579-1584

8. Gulani V, Calamante F, Shellock FG, Kanal E, Reeder SB (2017) Gadolinium deposition in the brain: summary of evidence and recommendations. Lancet Neurol 16:564-570

9. Baltzer PAT, Bickel H, Spick C et al (2018) Potential of noncontrast magnetic resonance imaging with diffusion-weighted imaging in characterization of breast lesions: intraindividual comparison with dynamic contrast-enhanced magnetic resonance imaging. Invest Radiol 53:229-235

10. Trimboli RM, Verardi N, Cartia F, Carbonaro LA, Sardanelli F (2014) Breast cancer detection using double reading of unenhanced MRI including T1-weighted, T2-weighted STIR, and diffusionweighted imaging: a proof of concept study. AJR Am J Roentgenol 203:674-681

11. McDonald ES, Hammersley JA, Chou SH et al (2016) Performance of DWI as a rapid unenhanced technique for detecting mammographically occult breast cancer in elevated-risk women with dense breasts. AJR Am J Roentgenol 207:205-216

12. Shin HJ, Chae EY, Choi WJ et al (2016) Diagnostic performance of fused diffusion-weighted imaging using unenhanced or postcontrast T1-weighted MR imaging in patients with breast cancer. Medicine (Baltimore) 95:e3502

13. Bickelhaupt S, Laun FB, Tesdorff J et al (2016) Fast and noninvasive characterization of suspicious lesions detected at breast cancer x-ray screening: capability of diffusion-weighted MR imaging with MIPs. Radiology 278:689-697

14. Baltzer PA, Benndorf M, Dietzel M, Gajda M, Camara O, Kaiser WA (2010) Sensitivity and specificity of unenhanced MR mammography (DWI combined with T2-weighted TSE imaging, ueMRM) for the differentiation of mass lesions. Eur Radiol 20: $1101-1110$

15. Baltzer P, Mann RM, Iima M et al (2019) Diffusion-weighted imaging of the breast-a consensus and mission statement from the EUSOBI International Breast Diffusion-Weighted Imaging working group. Eur Radiol. https://doi.org/10.1007/s00330-01906510-3

16. Pinker K, Moy L, Sutton EJ et al (2018) Diffusion-weighted imaging with apparent diffusion coefficient mapping for breast cancer detection as a stand-alone parameter: comparison with dynamic contrast-enhanced and multiparametric magnetic resonance imaging. Invest Radiol 53:587-595

17. Tamura T, Murakami S, Naito K, Yamada T, Fujimoto T, Kikkawa $T$ (2014) Investigation of the optimal b-value to detect breast tumors with diffusion weighted imaging by 1.5 -T MRI. Cancer Imaging 14:11
18. Burdette JH, Elster AD (2002) Diffusion-weighted imaging of cerebral infarctions: are higher B values better? J Comput Assist Tomogr 26:622-627

19. Blackledge MD, Leach MO, Collins DJ, Koh DM (2011) Computed diffusion-weighted MR imaging may improve tumor detection. Radiology 261:573-581

20. Zhou J, Chen E, Xu H et al (2019) Feasibility and diagnostic performance of voxelwise computed diffusion-weighted imaging in breast cancer. J Magn Reson Imaging 49:1610-1616

21. Gatidis S, Schmidt H, Martirosian P, Nikolaou K, Schwenzer NF (2016) Apparent diffusion coefficient-dependent voxelwise computed diffusion-weighted imaging: an approach for improving SNR and reducing T2 shine-through effects. J Magn Reson Imaging 43:824-832

22. O'Flynn EA, Blackledge M, Collins D et al (2016) Evaluating the diagnostic sensitivity of computed diffusion-weighted MR imaging in the detection of breast cancer. J Magn Reson Imaging 44: 130-137

23. Thomassin-Naggara I, De Bazelaire C, Chopier J, Bazot M, Marsault C, Trop I (2013) Diffusion-weighted MR imaging of the breast: advantages and pitfalls. Eur J Radiol 82:435-443

24. Dorrius MD, Dijkstra H, Oudkerk M, Sijens PE (2014) Effect of b value and pre-admission of contrast on diagnostic accuracy of 1.5-T breast DWI: a systematic review and meta-analysis. Eur Radiol 24: 2835-2847

25. Kul S, Metin Y, Kul M, Metin N, Eyuboglu I, Ozdemir O (2018) Assessment of breast mass morphology with diffusion-weighted MRI: Beyond apparent diffusion coefficient. J Magn Reson Imaging 48:1668-1677

26. D’Orsi C, Sickles EA, Mendelson EB, Morris EA (2013) Breast imaging reporting and data system: ACR BI-RADS breast imaging atlas, 5th edn. American College of Radiology, Reston, Va

27. Hricak H, Gatsonis C, Coakley FV et al (2007) Early invasive cervical cancer: CT and MR imaging in preoperative evaluation ACRIN/GOG comparative study of diagnostic performance and interobserver variability. Radiology 245:491-498

28. Xiao-Hua Zhou NAO, McClish DK (2011) Statistical methods in diagnostic medicine, 2nd edn. Wiley

29. Gonen M (2011) Analyzing receiver operating characteristic curves with SAS. SAS Institute, Cary, NC

30. Landis JR, Koch GG (1977) The measurement of observer agreement for categorical data. Biometrics 33:159-174

31. Partridge SC, Demartini WB, Kurland BF, Eby PR, White SW, Lehman CD (2010) Differential diagnosis of mammographically and clinically occult breast lesions on diffusion-weighted MRI. J Magn Reson Imaging 31:562-570

32. Avendano D, Marino MA, Leithner D et al (2019) Limited role of DWI with apparent diffusion coefficient mapping in breast lesions presenting as non-mass enhancement on dynamic contrastenhanced MRI. Breast Cancer Res 21:136

33. Tamura T, Takasu M, Higaki T et al (2019) How to improve the conspicuity of breast tumors on computed high b-value diffusionweighted imaging. Magn Reson Med Sci 18:119-125

34. Park JH, Yun B, Jang M et al (2019) Comparison of the diagnostic performance of synthetic versus acquired high b-value (1500 $\mathrm{s} / \mathrm{mm}(2))$ diffusion-weighted MRI in women with breast cancers. J Magn Reson Imaging 49:857-863

35. Cheng Q, Ye S, Fu C et al (2019) Quantitative evaluation of computed and voxelwise computed diffusion-weighted imaging in breast cancer. Br J Radiol 92:20180978

36. Bickel H, Polanec SH, Wengert G et al (2019) Diffusion-weighted MRI of breast cancer: improved lesion visibility and image quality using synthetic b-values. J Magn Reson Imaging 50:1754-1761

37. Chen X, He XJ, Jin R et al (2012) Conspicuity of breast lesions at different $\mathrm{b}$ values on diffusion-weighted imaging. BMC Cancer $12: 334$ 
38. Han X, Li J, Wang X (2017) Comparison and optimization of $3.0 \mathrm{t}$ breast images quality of diffusion-weighted imaging with multiple b-values. Acad Radiol 24:418-425

39. Mann RM, Kuhl CK, Moy L (2019) Contrast-enhanced MRI for breast cancer screening. J Magn Reson Imaging 50(2):377-390

40. Rosenkrantz AB, Hindman N, Lim RP et al (2013) Diffusionweighted imaging of the prostate: comparison of b1000 and b2000 image sets for index lesion detection. J Magn Reson Imaging 38:694-700

41. Maas MC, Futterer JJ, Scheenen TW (2013) Quantitative evaluation of computed high $\mathrm{B}$ value diffusion-weighted magnetic resonance imaging of the prostate. Invest Radiol 48:779-786

42. Moribata Y, Kido A, Fujimoto K et al (2017) Feasibility of computed diffusion weighted imaging and optimization of $b$-value in cervical cancer. Magn Reson Med Sci 16:66-72

43. Fukukura Y, Kumagae Y, Hakamada H et al (2017) Computed diffusion-weighted MR imaging for visualization of pancreatic adenocarcinoma: comparison with acquired diffusion-weighted imaging. Eur J Radiol 95:39-45
44. Woodhams R, Inoue Y, Ramadan S, Hata H, Ozaki M (2013) Diffusion-weighted imaging of the breast: comparison of bvalues $1000 \mathrm{~s} / \mathrm{mm}(2)$ and $1500 \mathrm{~s} / \mathrm{mm}(2)$. Magn Reson Med Sci 12:229-234

45. Partridge SC, DeMartini WB, Kurland BF, Eby PR, White SW, Lehman CD (2009) Quantitative diffusion-weighted imaging as an adjunct to conventional breast MRI for improved positive predictive value. AJR Am J Roentgenol 193:1716-1722

46. Ei Khouli RH, Jacobs MA, Mezban SD et al (2010) Diffusionweighted imaging improves the diagnostic accuracy of conventional 3.0-T breast MR imaging. Radiology 256:64-73

47. Baltzer A, Dietzel M, Kaiser CG, Baltzer PA (2016) Combined reading of contrast enhanced and diffusion weighted magnetic resonance imaging by using a simple sum score. Eur Radiol 26: 884-891

Publisher's note Springer Nature remains neutral with regard to jurisdictional claims in published maps and institutional affiliations. 